\title{
Light Sterile Neutrino from extra dimensions and Four-Neutrino Solutions to Neutrino Anomalies
}

\author{
A. Ioannisian * and J. W. F. Valle \\ Instituto de Física Corpuscular - C.S.I.C. \\ Departamento de Física Teòrica - Univ. de València \\ Edificio Institutos de Paterna - Apartado de Correos 2085 - 46071, València, Spain \\ http://neutrinos.uv.es
}

\begin{abstract}
We propose a four-neutrino model which can reconcile the existing data coming from underground experiments in terms of neutrino oscillations, together with the hint from the LSND experiment and a possible neutrino contribution to the hot dark matter of the Universe. It applies the idea that extra compact dimensions, probed only by gravity and possibly gauge-singlet fields, can lower the fundamental scales such as the Planck, string or unification scales. Our fourth light neutrino $\nu_{s}$ ( $s$ for sterile) is identified with the zero mode of the Kaluza-Klein states. To first approximation $\nu_{s}$ combines with the $\nu_{\mu}$ in order to form a Dirac neutrino with mass in the eV range leaving the other two neutrinos massless. The smallness of this mass scale (suitable for LSND and Hot Dark Matter) arises without appealing neither to a see-saw mechanism nor to a radiative mechanism, but from the volume factor associated with the canonical normalization of the wave-function of the bulk field in the compactified dimensions. On the other hand the splitting between $\nu_{\mu}$ and $\nu_{s}$ (atmospheric scale) as well as the mass of the two other neutrinos (solar mass scale) arise from the violation of the fermion number on distant branes. We also discuss alternative scenarios involving flavour-changing interactions. In one of them $\nu_{e}$ can be in the electron-volt range and therefore be probed in beta decay studies.
\end{abstract}

\footnotetext{
*On leave from Yerevan Physics Institute, Alikhanyan Br.2, Yerevan, 375036, Armenia.
} 


\section{INTRODUCTION}

It was recently suggested [1] that the hierarchy problem (the smallness of the weak scale to the Planck scale) may be avoided by simply removing the large scale. The observed small value of gravitational constant at long distance is ascribed to the spreading of the gravitational force in $n$ extra spatial dimensions. The relation between the scales where gravity becomes strong in the $4+\mathrm{n}$ dimensional theory can be derived from Gauss law

$$
M_{P l}^{2} \simeq\left(R M_{F}\right)^{n} M_{F}^{2}
$$

where $R$ is the compactification radius of the additional dimensions and $M_{F}$ is the fundamental Planck scale, which can be low. In what follows we take $M_{F} \simeq 10 \mathrm{TeV}$ and $n=6$ for which the corresponding value of $R$ is $R \simeq 10^{-12} \mathrm{~cm}$. It follows that the Standard Model (SM) fields (quarks, leptons, gauge fields and possible Higgs multiplets) are confined to a brane configuration [5], while the large compactified dimensions are probed only by gravity and bulk fields [2], singlet under the $S U(3) \otimes S U(2) \otimes U(1)$ gauge group. It has been recently shown [5] that this framework can be embedded into string models, where the fundamental Planck scale can be identified with the string scale which could be as low as the weak scale. The extra dimensions have the potential to lower the unification scale as well [6].

In Refs. [7,8] it was shown how neutrinos can naturally get very small Dirac masses via mixing with a bulk fermion. The main idea is that the coupling of the bulk fermions to the ordinary neutrino $\nu$ is automatically suppressed by a volume factor corresponding to the extra compactified dimension. Such a volume factor arises from the canonical normalization of the wave-function of the bulk field in the compactified dimensions. This volume factor provides a natural mechanism for suppressing the Yukawa coupling and correspondingly yields a light neutrino mass. In these theories, the left-handed neutrinos as well as other standard model (SM) particles, are localized on a brane embedded in the bulk of the large extra space. In Refs. [8,9] it was shown how to generate small Majorana masses for neutrinos via strong breaking fermion number on distant branes.

In this paper we study the implications of theories with extra dimensions with a few $\mathrm{TeV}$ scale of quantum gravity for neutrino physics. In contrast with other attempts to study neutrino masses and oscillations in models with large extra dimensions [10] we consider the possibility of using these ideas in order to find possible realistic ways to account for all the present neutrino observations from underground experiments $\mathrm{f}$,

\footnotetext{
${ }^{1}$ For a recent updated global analysis of solar and atmospheric neutrino data including the
} 
including both solar and atmospheric data, in terms of neutrino oscillations, in addition to the accelerator data, namely the possible hint from the LSND experiment. The latter requires at least one light sterile neutrino, in addition to the three (active) neutrinos. We propose four-neutrino models which explain the smallness of neutrino masses by exploiting the above mechanisms. In our model the sterile neutrino survives from the neutral fermions of the bulk sector, being identified with the zero mode of the KaluzaKlein states. Thus we bypass the need for a protecting symmetry to justify its lightness. To first approximation the sterile neutrinos and one of the active ones combine to form a Dirac state, while the other two remain massless. Next, as a result of fermion number breaking on distant branes, the heavier states split into a Quasi-Dirac state [15] at the eV scale, while the others get a small mass. Our simplest scheme obtained this way reproduces the model proposed in ref. [16], with the atmospheric neutrino data accounted for due to maximal mixing $\nu_{\mu}$ to $\nu_{s}$ oscillations, while the solar neutrino data is explained through $\nu_{e}$ to $\nu_{\tau}$ MSW conversions.

Since, although allowed [14], the $\nu_{\mu}$ to $\nu_{s}$ oscillation channel is not the preferred explanation of the atmospheric neutrino anomaly, we also briefly discuss the role of flavour-changing (FC) transitions. In this context we also mention, for example, a new variant of the model given in ref. [17] which is, however, physically inequivalent. In this new model the Quasi-Dirac neutrino with mass at the LSND scale combines $\nu_{e}$ with the sterile neutrino $\nu_{s}$, leaving $\nu_{\mu}$ and $\nu_{\tau}$ massless to first approximation. As a result the model leads to possible effects in tritium beta decay and the atmospheric neutrino data are well explained due to $\nu_{\mu}$ to $\nu_{\tau}$ conversions. In contrast to the first, this second scenario may successfully explain the solar neutrino data, but not via the MSW mechanism: it requires the presence of other physical processes such as flavourchanging (FC) transitions. These have been considered both in the context of solar neutrinos, as well of atmospheric neutrinos [18].

\section{THE SIMPLEST MODEL}

Here we concentrate in the minimum brane-inspired scheme in which all elements required to explain the neutrino anomalies (the LSND/HDM as well as the solar and atmospheric mass scales) are generated by the physics of extra dimensions. For definiteness, we will be considering a model that minimally extends the standard field content by one bulk neutrino, $N(x, y)$, singlet under the $S U(3) \otimes S U(2) \otimes U(1)$ gauge

solar neutrino recoil electron day and night spectra (for possible seasonal effects see [11]), as well as the atmospheric up-going muon data see ref. [12]. For previous refs. see ref. [13] and ref. [14. 
group. This propagates on a $[1+(3+\delta)]$-dimensional Minkowski space with $\delta \leq n$. Each $y$-coordinate of the large dimensions is compactified on a circle of radius $R$, by applying the periodic identification: $y \equiv y+2 \pi R$. Furthermore, we consider that only one four-dimension $S U(3) \otimes S U(2) \otimes U(1)$ singlet $\xi(x, y)$ from the higher-dimensional spinor $N(x, y)$ has non-vanishing Yukawa couplings, $\bar{h}_{l}$, to the three ordinary isodoublet leptons $L_{l}(x)$, with $l=e, \mu, \tau$. The relevant Lagrangian has the form

$$
\mathcal{L}_{\text {eff }}=\int d y^{\delta}\left[\bar{N}\left(i \gamma^{\mu} \partial_{\mu}+i \gamma_{\vec{y}} \partial_{\vec{y}}\right) N+\delta(y)\left(\sum_{l=e, \mu, \tau} \bar{h}_{l} L_{l} \tilde{\Phi} \xi+\text { H.c. }\right)+\delta(y) \mathcal{L}_{\mathrm{SM}}\right]
$$

where $\tilde{\Phi}=i \sigma_{2} \Phi^{*}$ and $\mathcal{L}_{\mathrm{SM}}$ describes the SM Lagrangian. The dimensionful Yukawa couplings $\bar{h}_{l}$ may be related to the dimensionless ones, $h_{l}^{(\delta)}$, through

$$
\bar{h}_{l}=\frac{h_{l}^{(\delta)}}{\left(M_{F}\right)^{\delta / 2}} .
$$

We can now express the $4+\delta$-dimensional two-component spinor $\xi$ in terms of a Fourier series expansion as follows:

$$
\xi(x, y)=\frac{1}{(2 \pi R)^{\frac{\delta}{2}}} \sum_{\vec{n}} \xi_{\vec{n}}(x) \exp \left(\frac{i \vec{n} \cdot \vec{y}}{R}\right)
$$

Substituting Eq. (4) into the effective Lagrangian (2) then performing the $y$ integrations and integrating out heavy Kaluza-Klein states yields

$$
\mathcal{L}_{\text {eff }}=\mathcal{L}_{\mathrm{SM}}+\left(\sum_{l=e, \mu, \tau} h_{l} L_{l} \tilde{\Phi} \xi_{0}+\text { H.c. }\right)
$$

where $\xi_{0}$ is the zero mode of the Kaluza-Klein states and

$$
h_{l}=\left(\frac{M_{F}}{M_{\mathrm{P}}}\right)^{\frac{\delta}{n}} h_{l}^{(\delta)} .
$$

As was first noticed in [7, 8], the four-dimensional Yukawa couplings $h_{l}$ are naturally suppressed by the volume factor $\left(\frac{M_{F}}{M_{\mathrm{P}}}\right)^{\frac{\delta}{n}}$ of the extra dimensions. With this we can estimate the effective four-dimensional Yukawa coupling and the corresponding neutrino mass in our model. In order to account for the LSND or hot dark matter mass scale $m_{\nu} \sim 1 \mathrm{eV}$ or so, we choose $\delta=4$ and $n=6$, giving $h_{l} \sim 10^{-10} h_{l}^{(\delta)}$.

Now we turn to the Majorana masses for neutrinos. These are crucial in order to generate the mass splittings required in neutrino oscillation interpretations of the solar and atmospheric neutrino anomalies found in underground experiments. As shown in Refs. [8,9] the neutrinos may get small Majorana masses via interactions with distant branes where fermion number is maximally broken. In case it is assumed that these interactions proceed via a very light field (lighter than $\sim 1 / R$ but heavier than $\sim$ $(\mathrm{mm})^{-1}$ to have escaped detection) which propagates in $4+\delta$ dimensions and that the 
brane where lepton number is broken is as far away as possible i. e. at a distance $\sim R$. The Majorana part of the neutrino masses is then expected to be

$$
m_{l l^{\prime}} \sim f_{l l^{\prime}} \frac{v^{2}}{M_{F}}\left(\frac{M_{F}}{M_{\mathrm{pl}}}\right)^{2 \delta / n-4 / n}
$$

The neutrino mass matrix takes in the basis $\left(\nu_{e}, \nu_{\mu}, \nu_{\tau}, \nu_{s}\right)\left(\nu_{s}=\xi_{0}\right)$ the form

$$
\mathcal{M}_{\nu}=\left(\begin{array}{cc}
m_{l l^{\prime}} & M_{l} \\
M_{l^{\prime}}^{T} & 0
\end{array}\right) .
$$

Here $M_{l}=h_{l} v$, where $v=174 \mathrm{GeV}$ is the standard electroweak vacuum expectation value. Note that the $m_{s}$ entry in eq. (8) has been omitted 1 since the bulk sector where the sterile neutrino $\nu_{s}$ lives is eight-dimensional (see Sec.2). There is, however, a general theorem which states that in eight dimensions there can be no massive Majorana spinor [19].

In the limit that Dirac mass terms $\left(M_{l}\right)$ are much bigger then Majorana mass terms $\left(m_{l l^{\prime}}\right)$ two of the neutrinos are massless and other two form Dirac state with a mass

$$
m \equiv m_{L S N D / H D M}=\sqrt{M_{e}^{2}+M_{\mu}^{2}+M_{\tau}^{2}}
$$

This state is identified by two angles $\theta$ and $\varphi$ defined as

$$
\sin \theta=\frac{M_{e}}{m}, \quad \tan \varphi=\frac{M_{\mu}}{M_{\tau}}
$$

The entries $m_{l l^{\prime}}$ only arise due to the breaking lepton number on distant branes. In the case $\delta=4$ and $n=6$ they are suppressed compared to the Dirac mass terms by the factor $\frac{v}{M_{F}} \frac{f_{l l^{\prime}}}{h_{l}}$. These terms give masses to the lowest-lying neutrinos and also responsible for splitting Dirac state to two Majorana states. For suitable values of the parameters, these are in the right range to have a solution for solar and atmospheric neutrino deficit. More especifically, from the latest fits one needs [14]

$$
\Delta m_{a t m}^{2} \simeq 3.5 \times 10^{-3} \mathrm{eV}^{2}
$$

${ }^{2}$ Note that one would have to add a term

$$
\left(\begin{array}{cc}
0 & M^{\prime} \\
M^{\prime} & M_{F}
\end{array}\right) .
$$

in the basis $\left(\nu_{s}, \chi\right)$, where $\chi$ denotes a fermion on a distant brane which couples with our bulk field $\nu_{s}$. Here $M_{F} \sim \mathrm{TeV}$ is the effective Planck mass and $M^{\prime} \sim \mathrm{eV}$ is suppressed by the same volume factor as $M_{l}$. Thus this would lead to an effective $m_{s}$ entry in eq. (8) of order $10^{-12} \mathrm{eV}$. Clearly this is totally irrelevant for our problem. 
in order to account for the full set of atmospheric neutrino data.

On the other hand the latest global analysis of solar neutrino data slightly prefers the large mixing MSW solution (LMA) characterized by the best-fit point 13

$$
\Delta m_{L M A}^{2} \simeq 3.6 \times 10^{-5} \mathrm{eV}^{2}
$$

Now assuming naturalness, namely, that masses and splittings are of the same order $\Delta m_{a t m} \simeq m_{l l^{\prime}} \simeq \sqrt{\Delta m_{\odot}^{2}}$ and since $\Delta m_{a t m}^{2} \simeq 2 m \Delta m_{a t m}$, one finds

$$
m \simeq 0.8 \mathrm{eV}
$$

characterizing the order of magnitude of the LSND/HDM scale in the LMA case.

On the other hand, for the SMA solution we have

$$
\Delta m_{S M A}^{2} \simeq 5 \times 10^{-6} \mathrm{eV}^{2}
$$

so that if $\Delta m_{S M A}^{2} \simeq(\Delta m)^{2}$ and using again $\Delta m_{a t m}^{2} \simeq 2 m \Delta m_{a t m}$ one finds

$$
m \simeq 0.3 e V
$$

characterizing the order of magnitude of the LSND/HDM scale in the SMA case.

Of course since clearly the solar mass splitting need not coincide exactly with the lightest state masses, the above estimates are meant to be crude order-of-magnitude estimates only. As a result the LSND/HDM scales both in the LMA and in the SMA case can be larger than estimated above.

In the above approximation the form of the charged current weak interaction may be given as

$$
-\frac{g}{\sqrt{2}} \sum_{i=1}^{3} \sum_{\alpha=1}^{4} \bar{e}_{i L} \gamma^{\mu} K_{i \alpha} \nu_{\alpha L}+\text { h.c. }
$$

where

$$
\mathcal{K}=\left(\begin{array}{cccc}
c_{\theta} c_{m} & c_{\theta} s_{m} & \frac{s_{\theta}}{\sqrt{2}} & \frac{s_{\theta}}{\sqrt{2}} \\
-s_{m} c_{\varphi}-s_{\theta} s_{\varphi} c_{m} & c_{\varphi} c_{m}-s_{\theta} s_{\varphi} s_{m} & \frac{c_{\theta} s_{\varphi}}{\sqrt{2}} & \frac{c_{\theta} s_{\varphi}}{\sqrt{2}} \\
s_{m} s_{\varphi}-s_{\theta} c_{\varphi} c_{m} & -s_{\varphi} c_{m}-s_{\theta} c_{\varphi} s_{m} & \frac{c_{\theta} c_{\varphi}}{\sqrt{2}} & \frac{c_{\theta} c_{\varphi}}{\sqrt{2}} \\
0 & 0 & -\frac{1}{\sqrt{2}} & \frac{1}{\sqrt{2}}
\end{array}\right)
$$

Here the first, second and third rows denote $\nu_{e}, \nu_{\mu}$ and $\nu_{\tau}$ respectively, while the fourth is the sterile neutrino $\nu_{s}$. The angles $\theta$ and $\varphi$ identify the dark matter neutrino while $\theta_{m}$ diagonalizes the light sector. The matrix $K$ determines also the structure of the neutral current weak interactions 20]

$$
-\frac{g^{\prime}}{2 \sin \theta_{W}} Z_{\mu} \sum_{\alpha \beta} \bar{\nu}_{L \alpha} \gamma_{\mu} P_{\alpha \beta} \nu_{L \beta}
$$

through the relation

$$
P=\mathcal{K}^{\dagger} \mathcal{K}
$$




\section{PHENOMENOLOGY}

If we assume that muonic neutrino coupling to the high dimensional spinor is dominant $\left(h_{e}, h_{\tau} \ll h_{\mu} \simeq 0.1\right)$ the light sterile neutrino, $\nu_{s}$, combines with $\nu_{\mu}$ and form a Quasi-Dirac state, crucial to account for the hint coming from the LSND experiment, and may also contribute to the hot dark matter of the Universe. Apart from the mass of the Quasi-Dirac state [15], one has the splittings between its components, as well as the masses of two light active states. The splittings between the heavy states and that characterizing the lighter neutrinos will be associated with the explanations of the atmospheric and solar neutrino anomalies, respectively. The atmospheric neutrino deficit is ascribed to the $\nu_{\mu}$ to $\nu_{s}$ oscilations. The solar neutrino problem could be solved via MSW small or large angle $\nu_{e}$ to $\nu_{\tau}$ solutions. This reproduces exactly the phenomenological features of the model proposed in ref. [16, providing a complete scenario for the present neutrino anomalies.

From eq.(17) we can determine the pattern of neutrino oscillations predicted in the model. In vacuo the neutrino oscillation probabilities are simply given as due to the cumulative non-decoupling effects of the Kaluza-Klein neutrinos in electroweak processes

$$
\begin{aligned}
& P\left(\nu_{e} \rightarrow \nu_{\mu}\right)=\sin ^{2} 2 \theta \sin ^{2} \varphi \quad \sin ^{2} \frac{m^{2} L}{4 E} \\
& P\left(\nu_{e} \rightarrow \nu_{\tau}\right)=\sin ^{2} 2 \theta \cos ^{2} \varphi \quad \sin ^{2} \frac{m^{2} L}{4 E} \\
& P\left(\nu_{\mu} \rightarrow \nu_{\tau}\right)=\sin ^{2} 2 \varphi \cos ^{4} \theta \quad \sin ^{2} \frac{m^{2} L}{4 E}
\end{aligned}
$$

One sees that the first consequence of our model is to have potentially detectable rates for neutrino oscillations in the laboratory. The LSND experiment gives the following constraints $\sin ^{2} 2 \theta \sin ^{2} \varphi \simeq 0.003$ (0.03) for $m \simeq 1.0(0.5) \mathrm{eV}$. On the other hand the Chooz experiment requires $\sin ^{2} 2 \theta \leq 0.18$ for $m \sim 0.5-1 \mathrm{eV}$.

Note that the $\mathrm{eV}$ range sterile neutrino range with maximal mixing with the active state predicted in our scenario would enter into equilibrium with the active neutrinos via neutrino oscillations in the early Universe [21], leading to an effective equivalent number of four light neutrinos in the early Universe, $N_{\nu}^{e f f}=4$. For recent discussions see ref. [22,23] and for a discussion of the possible role of lepton asymmetries see ref. [24]. This contrasts with the situation found in the model of ref. [17], since there the lightness of the sterile neutrino suppresses these conversions leading to an effective light neutrino number of three, $N_{\nu}^{e f f}=3$. The difference lies in that in the present case the Quasi-Dirac state (of mass $m \sim \mathrm{eV}$, for $M_{F} \simeq 10 \mathrm{TeV}$ ) inescapably includes the sterile neutrino, while in [17] the Quasi-Dirac state combines the two active $\nu_{\mu}$ and $\nu_{\tau}$ [25] leaving the $\nu_{s}$ in the lighter sector. 
Finally, note that the low value for the scale $M_{F} \simeq$ few $\mathrm{TeV}$ might lead to flavourviolation and universality-breaking phenomena potentially accessible to experiment due to the cumulative non-decoupling effects of the Kaluza-Klein neutrinos in electroweak processes, even though the original Yukawa couplings are small [26]. The rates of the flavour-violating decays $\mu \rightarrow e \gamma$ and $\mu \rightarrow$ eee as well as $\mu \rightarrow e$ coherent conversion in nuclei are proportional to the Yukawa couplings

$$
h_{e}^{2} h_{\mu}^{2}=\frac{m^{4}}{4 v^{4}} \sin ^{2} 2 \theta \sin ^{2} \varphi
$$

On the other hand the $\nu_{\mu} \rightarrow \nu_{e}$ oscillation probability eq. (20) depends on the same parameters. Fitting for the LSND experiment and using the upper limits from nonobservation of lepton flavour-violating and universality-breaking phenomena involving the $\mathrm{W}$ and $\mathrm{Z}$ bosons one obtains [26] the following restriction on the compactification scale $M_{F}$ in our model

$$
M_{F} \gtrsim 2 \mathrm{TeV}\left(\frac{m}{\mathrm{eV}}\right)^{3 / 5}\left(\frac{\sin ^{2} 2 \theta \sin ^{2} \varphi}{0.003}\right)^{3 / 20}
$$

Note that in the region indicated by the LSND experiment the product of the two terms in parenthesis in the right hand side are essentially constant and close to unity. With this one can obtain a lower bound on the scale $M_{F}, M_{F} \gtrsim 2 \mathrm{TeV}$. Note that this is an order of magnitude estimate only, since we do not know where to cut the Kaluza-Klein tower of the sterile neutrinos.

There are other conceivable brane-inspired four-neutrino scenarios capable of explaining of the solar and atmospheric neutrino problems. For example, if we assume that the electronic neutrino coupling to the high dimensional spinor is the dominant one $\left(h_{\mu}, h_{\tau} \ll h_{e} \simeq 0.1\right)$ then the Quasi-Dirac state combines $\nu_{e}$ with $\nu_{s}$. This leads to the possibility of observing neutrino mass effects in tritium beta decay experiments and also the $\nu_{e}$ would form part of the hot dark matter. The explanation of atmospheric neutrino deficit comes due to the $\nu_{\mu}$ to $\nu_{\tau}$ oscillations, which gives an excellent fit of the data [14, better than that for the $\nu_{\mu}$ to $\nu_{s}$ case. In contrast, the solar neutrino problem can not be accounted for in the framework of the MSW effect, since in this case we have large mixing angle $\nu_{e}$ to $\nu_{s}$ oscillations, which is ruled out by the solar data fit [13]. A possible way out is to assume the presence of other physical processes, such as flavour-changing (FC) transitions. In this case one may have fermion number violation on distant branes in such a way that only the $\nu_{\mu}$ and $\nu_{\tau}$ masses are split, but not the heavier neutrinos $\nu_{e}$ and $\nu_{s}$. This can provide an explanation of the solar neutrino data in the presence of flavour-changing neutral currents 18 . 


\section{DISCUSSION}

We have proposed a four-neutrino model which can explain the smallness of neutrino masses without appealing neither to a see-saw mechanism nor to a radiative mechanism. It applies the idea that extra compact dimensions, probed only by gravity and gaugesinglet fields, can lower the fundamental scales such as the Planck, string or unification scales. The light sterile neutrino $\nu_{s}$ is identified with the zero mode of the Kaluza-Klein states and combines with $\nu_{\mu}$ in order to form a Quasi-Dirac neutrino with mass in the $\mathrm{eV}$ range, contributing to the hot dark matter of the Universe. Apart from the possible effect of lepton asymmetries the sterile neutrinos are brought into equilibrium in the early Universe, leading to four effective light neutrinos $N_{\nu}^{e f f}=4$. Fermion number breaking effects on distant branes lead to the splitting of this Quasi-Dirac state as well as two massless active states. These splittings generate oscillations which may reconcile the existing data coming from underground experiments with the hint from LSND. The simplest scheme is the same as the one proposed in ref. [16], accounting for the atmospheric data in terms of $\nu_{\mu}$ to $\nu_{s}$ oscillations and the solar neutrino data in terms of $\nu_{e}$ to $\nu_{\tau}$ MSW conversions.

The scheme can be tested through lepton flavour violating processes such as $\mu \rightarrow$ $e+\gamma, \mu \rightarrow 3 e$ and muon conversion in nuclei. In particular we showed that fitting for the LSND signal enables us to estimate the muon-number violating rates as a function of the fundamental Planck scale, $M_{F}$ which must exceed few $\mathrm{TeV}$ or so. Finally, note that in our scheme there is no expected modification of the Newtonian law accessible at the proposed sub-millimeter scale.

We have also discussed a second possible model which accounts for the atmospheric data in terms of $\nu_{\mu}$ to $\nu_{\tau}$ oscillations. However, in contrast to the original model of ref. [17], such model is incompatible with an explanation of the solar data in the framework of the simplest MSW effect since the large mixing predicted between $\nu_{e}$ and $\nu_{s}$ is ruled out by the solar neutrino data 13 [5. This can be remedied in the presence of flavour-changing (FC) interactions.

Finally we comment on another recent attempt [27] to obtain a four-neutrino model based on extra dimensions. The model in ref. [27] is totally different. Ours is minimal in the sense that we have simply the standard $S U(3) \otimes S U(2) \otimes U(1)$ gauge group and no right-handed neutrinos, while they explain the lightness of the active neutrinos via the standard left-right symmetric see-saw mechanism. Moreover we have a much lower value for the fundamental Planck scale in our model, $M_{F} \sim 10^{4} \mathrm{GeV}$. This leads to a plethora of lepton flavour violating processes which are expected to be negligible in

\footnotetext{
${ }^{3}$ This follows from the behaviour of the neutrino conversion probability expected for large mixing combined with the absence of neutral currents in active-to-sterile conversions.
} 
the model of ref. [27] and which could potentially be used to distinguish them. Finally, in our case all light neutrino masses arise from the extra dimensions mechanisms: the $\mathrm{eV}$ scale follows from the volume factor associated with the canonical normalization of the wave-function of the bulk field in the compactified dimensions, while solar and atmospheric mass scales arise from the violation of the fermion number on distant branes.

\section{ACKNOWLEDGEMENT}

We thank Subir Sarkar and Sasha Dolgov for discussions on the present status of primordial nucleosynthesis bounds and their uncertainties. This work was supported by DGICYT grants PB98-0693 and SAB1998-0136, as well as the Alexander von Humboldt Foundation and the European Union, under TMR contract ERBFMRX-CT96-0090.

[1] I. Antoniadis, Phys. Lett. B246 (1990) 377.

[2] N. Arkani-Hamed, S. Dimopoulos, and G, Dvali, Phys. Let. B429, 263 (1998).

[3] I. Antoniadis, N. Arkani-Hamed, S. Dimopoulos, and G. Dvali, Phys. Lett. B436, 257 (1998) hep-ph/9804398.

[4] N. Arkani-Hamed, S. Dimopoulos, and G, Dvali, Phys. Rev. D59,086004 (1999).

[5] J. Lykkin, Talk at the International Workshop on Phenomenological Aspects of Superstring Theories (PAST97), Trieste, Italy,2-4 October, 1997.

[6] K. R. Dienes, E. Dudas, and T. Gherghetta, Phys. Lett. B436,55 (1998).

[7] K. R. Dienes, E. Dudas, and T. Gherghetta, hep-ph/9811428.

[8] N. Arkani-Hamed, S. Dimopoulos, G, Dvali, and John March-Russell, hep-ph/9811448.

[9] N. Arkani-Hamed, S. Dimopoulos, hep-ph/9811353.

[10] For a recent paper see A. Yu. Smirnov, proceedings of International Workshop on Particles in Astrophysics and Cosmology: From Theory to Observation, Valencia, Spain, May 3-8, 1999, Nuclear Physics B, Proceedings Supplements, 2000, Vol. 81, ISSN 0920-5632, Ed. V. Berezinsky, G. Raffelt and J. W. F. Valle; R.N. Mohapatra, S. Nandi and A. PerezLorenzana, hep-ph/9907520; A. Das and O.C. Kong, hep-ph/9907272; G.C. McLaughlin and J.N. Ng, hep-ph/9909558.

[11] P.C. de Holanda, C. Pena-Garay, M.C. Gonzalez-Garcia and J.W. Valle, Phys. Rev. D60, 
093010 (1999), hep-ph/9903473, see also J.N. Bahcall, P.I. Krastev and A.Y. Smirnov, hep-ph/9905220.

[12] M. C. Gonzalez-Garcia, M. Maltoni, C. Pena-Garay and J. W. Valle, hep-ph/0009350, Phys. Rev. D, in press.

[13] M.C. Gonzalez-Garcia, P.C. de Holanda, C. Pena-Garay and J.W. F. Valle, Nucl. Phys. B573, 3 (2000) [hep-ph/9906469.

[14] N. Fornengo, M. C. Gonzalez-Garcia and J. W. Valle, "Updated global analysis of the atmospheric neutrino data in terms of neutrino oscillations," Nucl. Phys. B580 (2000) 58 hep-ph/0002147. For previous discussions see, e.g. M.C. Gonzalez-Garcia, et al Nucl. Phys. B543, 3 (1999), hep-ph/9807305, and of Phys. Rev. D58, 033004 (1998), hep$\mathrm{ph} / 9801368$.

[15] J. W. F. Valle, Phys. Rev. D27 (1983) 1672; for the alternative case of Quasi Dirac Neutrino made up of two active components see [25].

[16] J. T. Peltoniemi, D. Tommasini, and J. W. F. Valle, Phys. Lett. B298, 383 (1993)

[17] J. Peltoniemi and J. W. F. Valle, Nucl.Phys. B406, 409 (1993); D. Caldwell and R. Mohapatra, Phys. Rev. D48, 3259 (1993); for more phenomenological discussions see S. Bilenky, C. Giunti and W. Grimus, Eur. Phys. J. C1, 247 (1998) and M. Hirsch and J. W. Valle, "Reconciling neutrino anomalies in a simple four-neutrino scheme with R-parity violation," hep-ph/0009066, to be be published in Phys. Lett. B.

[18] Both solar and atmospheric neutrino data may be accounted for by FC neutrino interactions. For example the atmospheric data have been recently considered in N. Fornengo, M. C. Gonzalez-Garcia and J. W. F. Valle, JHEP 0007 (2000) 006 hep-ph/9906539; M. C. Gonzalez-Garcia et al., Phys. Rev. Lett. 82, 3202 (1999) hep-ph/9809531. For a recent and detailed study of the solution to the solar neutrino problem based on non-standard neutrino interactions, see S. Bergmann, M. M. Guzzo, P. C. de Holanda, P. I. Krastev and H. Nunokawa, hep-ph/0004049.

[19] J. Scherk, Recent Developments in Gravitation, Cargese Summer Institute, 1978, ed. M. Levy and S. Deser (Plenum, NY, 1979), page 179; S. Weinberg, Phys. Lett. B143, 97 (1984); K. Shimizu, Prog. Theor. Phys. 74, 610 (1985); C. Wetterich, Nucl. Phys. B234, 413 (1984); R. Finkelstein and M. Villasante, Phys. Rev. D31, 425 (1985); A. Pilaftsis, Phys. Rev. D60, 105023 (1999) hep-ph/9906265.

[20] J. Schechter and J .W. F. Valle, Phys. Rev. D22, 2227 (1980).

[21] R. Barbieri and A. Dolgov, Phys. Lett. B 237, 440 (1990); K. Enquist, K. Kainulainen and J. Maalampi, Phys. Lett. B 249, 531 (1992); D. P. Kirilova and M. Chizov, hep$\mathrm{ph} / 9707282$. 
[22] S. Sarkar, Rep. Prog. Phys. 59, 1493 (1996); P. J. Kernan and S. Sarkar, Phys. Rev. D 54 (1996) R3681

[23] G. Fiorentini, E. Lisi, S. Sarkar and F.L. Villante, Phys. Rev. D58, 063506 (1998);

E. Lisi, S. Sarkar and F.L. Villante, Phys. Rev. D59, 123520 (1999)

[24] R. Foot, R.R. Volkas, Phys. Rev. D55 (1997) 5147-5176; A.D. Dolgov, S.H. Hansen, S. Pastor and D.V. Semikoz, hep-ph/9910444.

[25] L. Wolfenstein, Nucl. Phys. B186 (1981) 147; J .W. F. Valle and M. Singer, Phys. Rev. D28 (1983) 540.

[26] A. Ioannisian and A. Pilaftsis, hep-ph/9907522; see also A.E. Faraggi and M. Pospelov, Phys. Lett. B458 (1999) 237

[27] R. N. Mohapatra and A. Perez-Lorenzana, hep-ph/9910474. 